\title{
Improving the power efficiency of white light-emitting diode by doping electron transport material
}

\author{
Jinsong Huang, Wei-Jen Hou, Juo-Hao Li, Gang Li, and Yang Yang ${ }^{a)}$ \\ Department of Materials Science and Engineering, University of California-Los Angeles, Los Angeles, \\ California 90095-1595
}

(Received 14 July 2006; accepted 17 August 2006; published online 27 September 2006)

\begin{abstract}
Highly efficient white light emission was realized via the partial energy transfer from blue host polyfluorene $(\mathrm{PF})$ to orange light emission dopant rubrene. A more balanced charge transport was achieved by adding an electron transport material, 2-(4-biphenylyl)-5-(4-tertbutylphenyl)-1,3,4-oxadiazole (PBD), into the PF-rubrene system to enhance the electron transportation. Efficiency improvement by as much as a factor of 2 has been observed through the addition of PBD. These devices can easily reach high luminance at low driving voltages, thus achieving high power efficiency at high luminance $(14.8,13.5$, and $12.0 \mathrm{~lm} / \mathrm{W}$ at the luminances of 1000,2000 , and $4000 \mathrm{~cd} / \mathrm{m}^{2}$, respectively). Therefore, this performance is an important approach toward solid-state lighting application. The enhancement is mainly attributed to three factors: increased electron transport property of the host material, increased photoluminescence quantum efficiency, and the shifting of emission zone away from cathode contact. The reported efficiency is among the highest values reported in the white emission polymer light-emitting diodes. (C) 2006 American Institute of Physics. [DOI: 10.1063/1.2357938]
\end{abstract}

White light-emitting diodes based on organic (WOLEDs) or polymer (WPLEDs) materials have been intensively studied due to their potential in next generation solid-state lighting. ${ }^{1-10}$ In particular, solution process (often found in polymer coating) provides a low-cost approach for this application. In the past, the efficiency in WPLEDs was generally lower than that of WOLEDs due to unbalanced charges and impurities in polymer. A recent breakthrough in fluorescent WPLEDs, however, enables the WPLED performance to exceed that of incandescent lamps. ${ }^{7}$ In such devices, a nanoscale cesium carbonate $\left(\mathrm{Cs}_{2} \mathrm{CO}_{3}\right)$ layer, serving dual purposes of electron injection and hole-blocking, is placed between the cathode and the light-emitting-polymer (LEP) layer. White light emission is achieved by a polymer blend consisting of polyfluorene $(\mathrm{PF})$ and poly[2-methoxy5-(2'-ethyl-hexyloxy)-1,4-phenylene vinylene] (MEH-PPV) and a partial energy transfer between them. In that example, although PF serves well for light emission and partial energy transfer, its transport property is rather imbalanced between holes and electrons. Time-of-flight measurements show that electron transport is much poorer than hole transport due to the presence of high density electron traps within the polymer. ${ }^{11}$ Since charge transport is mainly determined by the host material, it is crucial that the host material posseses an efficient and balanced charge transport property. In this letter, we report the doping of a well-known electron transport material, 2-(4-biphenylyl)-5-(4-tert-butylphenyl)-1,3,4oxadiazole (PBD), ${ }^{12}$ into PF to enhance its electron transport property.

MEH-PPV is a good candidate for the orange light emission dopant, and it can form a homogeneous blend with PF when the concentration of MEH-PPV is less than 4 wt \%. In this work, MEH-PPV was replaced with an orange emission small molecular material, rubrene, because it has been reported that rubrene is able to achieve photoluminescence quantum efficiency (PLQE) of nearly 100\% when it is doped

${ }^{a)}$ FAX: (310) 825-3665; electronic mail: yangy@ucla.edu into another host material. ${ }^{13}$ All of the devices discussed in this work are fabricated based on the structure of indium tin oxide/PEDOT:PSS/PF:PBD:rubrene/ $\mathrm{Cs}_{2} \mathrm{CO}_{3} / \mathrm{Al}$, PEDOT:PSS stands for poly(ethylenedioxythiophene):polystyrene sulfonate. The $\mathrm{Cs}_{2} \mathrm{CO}_{3}$ layer was spin cast from dilute solution of $0.2 \mathrm{wt} \% \mathrm{Cs}_{2} \mathrm{CO}_{3}$ in 2-ethoxyethanol. PEDOT:PSS, LEP, and $\mathrm{Cs}_{2} \mathrm{CO}_{3}$ were deposited by spin casting one layer after another. The spin-cast LEP film was under heat treatment at $70{ }^{\circ} \mathrm{C}$ for $30 \mathrm{~min}$ before the coating of $\mathrm{Cs}_{2} \mathrm{CO}_{3}$ layer. Finally, the fabrication of devices is completed by thermal deposition of $100 \mathrm{~nm} \mathrm{Al}$. All the processes starting from spin coating of LEP to the evaporation of $\mathrm{Al}$ cathode were carried out under nitrogen atmosphere. The concentration of rubrene is fixed at $0.2 \mathrm{wt} \%$ to obtain the white emission, while the concentration of PBD varies from 0 to $8 \mathrm{wt} \%$ to optimize the device performance.

Figure 1 shows the voltage $(V)$ dependence on current density $(J)$ and luminance $(L)$ for the devices with different PBD concentrations. Compared to the device without PBD doping, the PBD containing device has a sharper increase in current density. Both current and luminance increase monotonically as the concentration of PBD increases. A detailed examination of the $J-V$ characteristic shows an abrupt increase in current density between 5 and 8 wt \% PBD concentrations. Such effect can be explained by the formation of electron transport subnetwork in the host material. It is called subnetwork because it is not the strong network for electrons to percolate through the film. Otherwise all electrons will transport directly to anode via network and no electron/hole recombination will occur. Since PBD has comparable, if not higher, lowest unoccupied molecular orbital level $(2.4-2.6 \mathrm{eV}),{ }^{14,15}$ to that of PF $(2.1-2.8 \mathrm{eV}),{ }^{16}$ it is unlikely that PBD will help the electron injection from cathode to host material. Also, there is no report showing that PBD has good hole conduction property. PBD has electron mobility of $3 \times 10^{-3} \mathrm{~cm}^{2} /(\mathrm{V} \mathrm{s}),{ }^{17}$ which is several orders of magnitude larger than that of PF $\left[10^{-8}-10^{-7} \mathrm{~cm}^{2} /(\mathrm{V} \mathrm{s})\right] .{ }^{18}$ So the increased conduction property arises from the enhanced elec- 


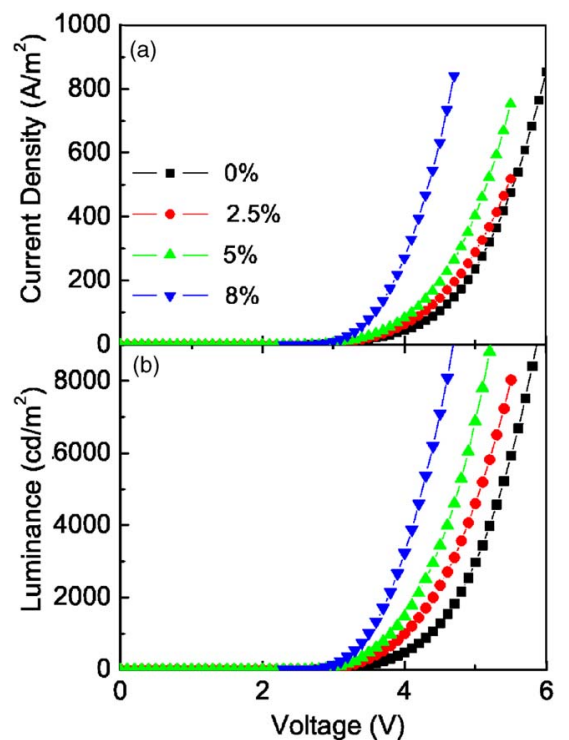

FIG. 1. (Color online) (a) $J-V$ and (b) $L-V$ characteristics for the devices with different PBD concentrations.

tron transport in the PF by the formation of electron transport network.

Figure 2 shows the luminous efficiency (a) and power efficiency (b) of devices at different PBD concentrations. The electroluminescence (EL) spectra of these devices at $25 \mathrm{~mA} / \mathrm{cm}^{2}$ are also shown in the inset of Fig. 2(a). The EL spectra are essentially unaffected by addition of PBD from 0 to $5 \mathrm{wt} \%$. However a distinct change in the EL spectra is observed when PBD concentration changes to 8 wt \%; the relative emission from rubrene becomes weaker as compared to the emission from PF. This change concurs with the abrupt increase in current density mentioned above when PBD concentration changes from 5 to $8 \mathrm{wt} \%$. This phenomenon can be understood by the following scenario: It is known that rubrene, as a dopant material, can act as a low energy site for energy transfer as well as a trapping site for charge trapping. The relative decrease in EL emission from rubrene can be explained by the decrease of trapped charges on rubrene, and this is due to the formation of electron transport network at higher PBD concentration. The device made of PF:rubrene blend has a peak luminous efficiency of $12.6 \mathrm{~cd} / \mathrm{A}$. With the increase of PBD concentration in PF, the peak luminous efficiency first increases to a maximum of $17.9 \mathrm{~cd} / \mathrm{A}$ at $5 \mathrm{wt} \%$ PBD concentration, and then drops down to $13.3 \mathrm{~cd} / \mathrm{A}$ at $\mathrm{PBD}$ concentration of $8 \mathrm{wt} \%$. Similar variation is also observed in the power efficiency at different PBD concentrations. The peak power efficiency increases from $8.8 \mathrm{~lm} / \mathrm{W}$ at $0 \mathrm{wt} \% \mathrm{PBD}$ to $16.3 \mathrm{~lm} / \mathrm{W}$ at $5 \mathrm{wt} \% \mathrm{PBD}$, and drops to $13.9 \mathrm{~lm} / \mathrm{W}$ at $8 \mathrm{wt} \% \mathrm{PBD}$. Luminous efficiency
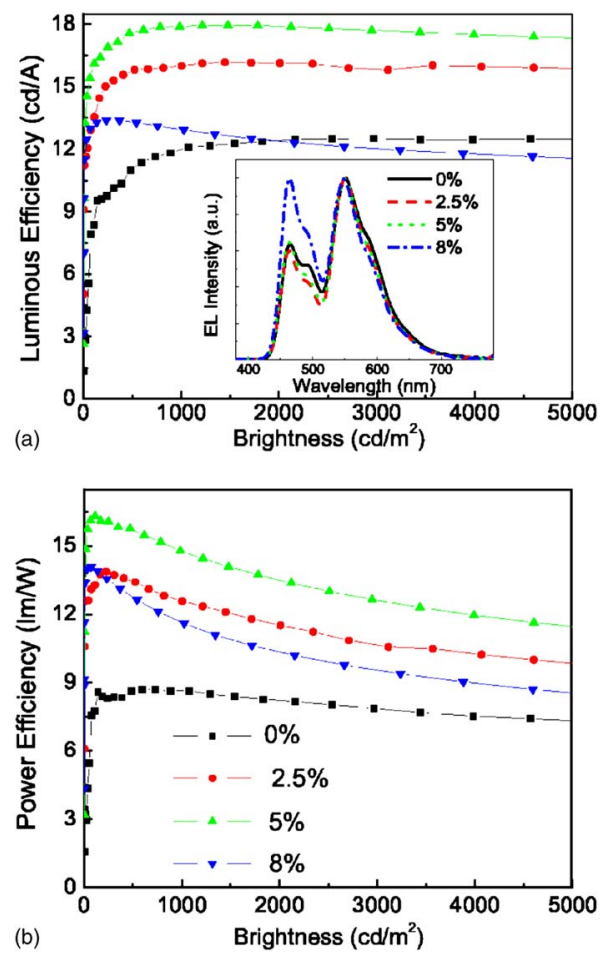

FIG. 2. (Color online) (a) Luminous efficiency and (b) power efficiency of devices with PBD concentrations varied from 0 to 8 wt \%. The inset of (a) shows EL spectra of the devices with different PBD concentrations at current density of $25 \mathrm{~mA} / \mathrm{cm}^{2}$.

increases by about $40 \%$ at 5 wt $\%$ PBD doping, while power efficiency is approximately doubled compared to the device without PBD doping. So it is easy to speculate that the increase of power efficiency comes from the two contributions: one is increased luminous efficiency and the other is lower driving voltage, which is obviously due to the improved electron transport property of host material by PBD doping. It is worth pointing out that these devices have even better performance than our previous devices based on blends of PF and MEH-PPV. ${ }^{7}$ Although they have similar maximum power efficiency, much higher power efficiencies at very high luminance can be obtained in these devices: for the $5 \mathrm{wt} \%$ PBD doping device, power efficiency at luminance of $3000 \mathrm{~cd} / \mathrm{m}^{2}$ is still as high as $12.6 \mathrm{~lm} / \mathrm{W}$, compared to the value of $8.9 \mathrm{~lm} / \mathrm{W}$ for a device based on MEH-PPV:PF blend. The performance of the devices is summarized in Table I.

It is essential from both device engineering and device physics perspectives to understand the mechanism governing the enhancement in luminous efficiency. There are several factors to be considered: injection efficiency, charge balance, emission zone location (outcoupling efficiency), and PLQE.

TABLE I. List of the performance of the devices with different PBD compositions.

\begin{tabular}{lcccc}
\hline \hline $\begin{array}{c}\text { PBD } \\
(\mathrm{wt} \%)\end{array}$ & $\begin{array}{c}\text { Driving voltage } \\
(\mathrm{V}) \text { at } 25 \\
\mathrm{~mA} / \mathrm{cm}^{2}\end{array}$ & $\begin{array}{c}\text { CIE coordinates } \\
\text { at } 25 \mathrm{~mA} / \mathrm{cm}^{2}\end{array}$ & $\begin{array}{c}\text { Luminous } \\
\text { efficiency } \\
(\mathrm{cd} / \mathrm{A})^{\mathrm{a}}\end{array}$ & $\begin{array}{c}\text { Power } \\
\text { efficiency } \\
(\mathrm{lm} / \mathrm{W})^{\mathrm{a}}\end{array}$ \\
\hline 0 & 5.03 & $(0.34,0.44)$ & $12.6 / 12.5$ & $8.8 / 7.8$ \\
2.5 & 4.87 & $(0.34,0.44)$ & $16.2 / 16.0$ & $13.9 / 10.6$ \\
5 & 4.64 & $(0.33,0.43)$ & $17.9 / 17.7$ & $16.3 / 12.6$ \\
8 & 3.94 & $(0.30,0.39)$ & $13.3 / 12.0$ & $14.0 / 9.5$ \\
\hline \hline
\end{tabular}

${ }^{\mathrm{a}}$ The former numbers are the maximum values, and the latter numbers are efficiency at the luminance of $3000 \mathrm{~cd} / \mathrm{m}^{2}$. 


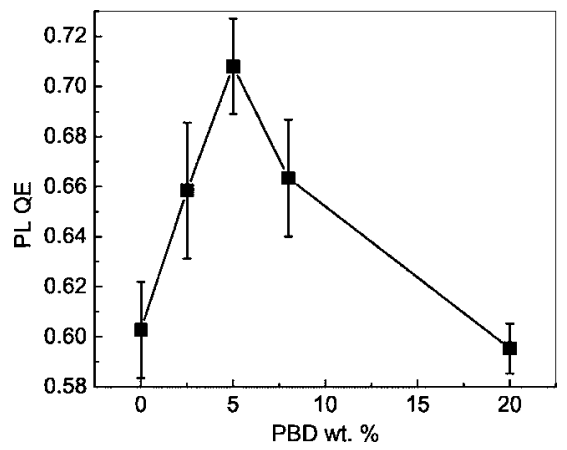

FIG. 3. PLQE of films at different PBD concentrations.

All factors are considered in our analysis. PLQE is measured for the films with different PBD concentrations using an integrating sphere from Labsphere Inc. according to the method described by Greenham et al. ${ }^{19}$ We use an UV excitation at $\lambda_{\text {exc }}=337 \mathrm{~nm}$, provided by a nitrogen laser. The photoluminescence from the exit port is coupled into a silicon charge coupled device (CCD) by an optical fiber. The CCD is calibrated together with the optical fiber. Although PBD is expected to partially absorb the excitation light and thus participate in the energy-transfer process of the blend system, we have not observed any changes in the PL spectra of films with different PBD concentrations, in contrast to EL spectra. Therefore, it is likely that the exciton lifetime of PBD is too short to cause any changes in the PL spectra. However, there are variations in the PLQE of films with different PBD concentrations. As shown in Fig. 3, the PLQE of PF:rubrene film reaches a maximum of $71 \%$ at $5 \mathrm{wt} \%$ doping concentration of PBD, and decreases with further increase in PBD concentration. The authors would like to point out that although a similar behavior in a $N, N^{\prime}$-diphenyl- $N, N^{\prime}$-(bis(3-methylphenyl)-[1,1-bipheny]-4,4' -diamine (TPD) doped into polyvinyl carbazole:PBD:Ir(ppy) ${ }_{3}$ system was previously reported by Kalinowski et al., ${ }^{20}$ the mechanisms governing both behaviors are entirely different. Since TPD has similar triplet level as $\operatorname{Ir}(\mathrm{ppy})_{3}$ but with longer triplet lifetime, it was believed that TPD functions as triplet exciton reservoir for $\operatorname{Ir}(\mathrm{ppy})_{3}$. On the other hand, PBD has much higher band gap than both $\mathrm{PF}$ and rubrene, therefore, it is energetically unfavorable for PBD to act as an energy reservoir for either host or guest material. We believe that the increase in PLQE at low concentration of PBD is attributed to the reduced interchain reaction (aggregation effect) in $\mathrm{PF}$, and the decrease in PLQE at higher PBD concentration is simply due to the inherent low PLQE of PBD material. A detailed investigation on the kinetics of the energy-transfer process is under way using time-resolved photoluminescence spectroscopy.

Since the effect of PBD concentration in PLQE (Fig. 3) behaves in the same way as the efficiency of the device (Fig. 2 ), the increase in luminous efficiency $(40 \%)$ is attributed in part to the increase in PLQE (20\%). The remaining contribution comes from a more balanced charge transport and the shift of emission zone away from the electrode. The increased electron transport property of the host will broaden the emission zone by shifting part of recombination toward the middle of LEP layer. And it has been proven both theoretically and experimentally that the outcoupling efficiency increases when the emission zone shifts from near cathode to the middle of LEP layer. ${ }^{8,21}$ One particular phenomenon accompanying shift of emission zone is the change of EL spectrum shape due to the microcavity effect. As one can see from the inset of Fig. 2(a), there is a small difference in the ratio between shoulder and main peak in EL spectra for each emission component when PBD concentration changes from 0 to $5 \mathrm{wt} \%$. Such difference cannot be found in the EL spectra at different driving voltages. And since the devices have the same parameters except for the PBD concentration, we expect that the difference in the EL spectra arises from the microcavity effect or shift of recombination zone.

In summary, we demonstrated improvement in power efficiency of WPLED by the doping of PBD into the PFrubrene system. The devices have high power efficiency at very high luminance, which brings them a bright future for the illumination application. PBD can improve the electron transport property of $\mathrm{PF}$, and in the meantime, increase the PLQE of the PF:PBD:rubrene polymer-small molecule blends. The shift of emission zone is also accounted for by the increased efficiency.

One of the authors (W.J.H.) acknowledges the financial support from NSF IGERT: Materials Creation Training Program (DGE-0114443).

${ }^{1}$ J. Kido, M. Kimura, and K. Nagai, Science 267, 1332 (1995).

${ }^{2}$ B. W. D'Andrade, R. J. Holmes, and S. R. Forrest, Adv. Mater. (Weinheim, Ger.) 16, 624 (2004).

${ }^{3}$ G. Li and J. Shinar, Appl. Phys. Lett. 83, 5359 (2003).

${ }^{4}$ Mitsunori Suzuki, Takuya Hatakeyama, Shizuo Tokito, and Fumio Sato, IEEE J. Sel. Top. Quantum Electron. 10, 115 (2004).

${ }^{5}$ C. H. Chuen and Y. T. Tao, Appl. Phys. Lett. 81, 4499 (2002).

${ }^{6}$ Xiong Gong, Shu Wang, Daniel Moses, Guillermo C. Bazan, and Alan H. Heeger, Adv. Mater. (Weinheim, Ger.) 17, 2053 (2005).

${ }^{7}$ Jinsong Huang, Gang Li, Elbert Wu, Qianfei Xu, and Yang Yang, Adv. Mater. (Weinheim, Ger.) 18, 114 (2006).

${ }^{8}$ S. Choulis, V. Choong, M. K. Mathai, and F. So, Appl. Phys. Lett. 87, 113503 (2005).

${ }^{9}$ J. Liu, Q. G. Zhou, Y. X. Cheng, Y. H. Geng, L. X. Wang, D. G. Ma, X. B. Jing, and F. S. Wang, Adv. Mater. (Weinheim, Ger.) 17, 2974 (2005).

${ }^{10}$ Guoli Tu, Chongyu Mei, Quanguo Zhou, Yanxiang Cheng, Yanhou Geng, Lixiang Wang, Dongge Ma, Xiabin Jing, and Fosong Wang, Adv. Funct. Mater. 16, 101 (2006).

${ }^{11}$ M. Redecker, D. D. C. Bradley, M. Inbasekaran, and E. P. Woo, Appl. Phys. Lett. 73, 1565 (1998).

${ }^{12}$ Chihaya Adachi, Tetsuo Tsutsui, and Shogo Saito, Appl. Phys. Lett. 55, 1489 (1989)

${ }^{13}$ Hedi Mattoussi, Hideyuki Murata, Charles D. Merritt, Yasuhiro Iizumi, Junji Kido, and Zakya H. Kafafi, J. Appl. Phys. 86, 2642 (1999).

${ }^{14}$ Jörn Pommerehne, Horst Vestweber, Werner Guss, Rainer F. Mahrt, Heinz Bässler, Michael Porsch, and Jörg Daub, Adv. Mater. (Weinheim, Ger.) 7, 551 (1995).

${ }^{15}$ Fushan Li, Zhijian Chen, Wei Wei, Huayu Cao, Qihuang Gong, Feng Teng, Lei Qian, and Yuanmin Wang, J. Phys. D 37, 1613 (2004).

${ }^{16}$ Alasdair J. Campbell, Donal D. C. Bradley, and Homer Antoniadis, J. Appl. Phys. 89, 3343 (2001); L. S. Liao, F. Cheng, M. K. Fung, C. S. Lee, S. T. Lee, M. Inbasekaran, E. P. Woo, and W. W. Wu, Phys. Rev. B 62, 10004 (2000); W. Ma, P. K. Iyer, X. Gong, B. Liu, D. Moses, G. C. Bazan, and A. J. Heeger, Adv. Mater. (Weinheim, Ger.) 17, 274 (2005).

${ }^{17}$ Minlu Zhang, Zhijun Hu, and Tianbai He, J. Phys. Chem. B 108, 19198 (2004).

${ }^{18}$ D. J. Pinner, R. H. Friend, and N. Tessler, J. Appl. Phys. 86, 5116 (1999).

${ }^{19}$ N. C. Greenham, I. D. W. Samuel, G. R. Hayes, R. T. Phillips, Y. A. R. R. Kessener, S. C. Moratti, A. B. Holmes, and R. H. Friend, Chem. Phys. Lett. 241, 89 (1995).

${ }^{20}$ J. Kalinowski, W. Stampor, M. Cocchi, D. Virgili, V. Fattori, and P. Di Marco, Chem. Phys. 297, 39 (2004).

${ }^{21}$ H. Riel, S. Karg, T. Beierlein, W. Rieß, and K. Neyts, J. Appl. Phys. 94, 5290 (2003). 